\title{
CONVERGENT SEMIDISCRETIZATION OF A NONLINEAR FOURTH ORDER PARABOLIC SYSTEM
}

\author{
Ansgar JÜngel ${ }^{1}$ AND René PinnaU ${ }^{2}$
}

\begin{abstract}
A semidiscretization in time of a fourth order nonlinear parabolic system in several space dimensions arising in quantum semiconductor modelling is studied. The system is numerically treated by introducing an additional nonlinear potential. Exploiting the stability of the discretization, convergence is shown in the multi-dimensional case. Under some assumptions on the regularity of the solution, the rate of convergence proves to be optimal.
\end{abstract}

Mathematics Subject Classification. 35K35, 65M12, 65M15, 65M20, 76Y05.

Received: May 29, 2002. Revised: November 20, 2002.

\section{INTRODUCTION}

During the last decade the interest in constructing positivity-preserving numerical schemes for fourth order nonlinear parabolic equations grew significantly $[3,8,20]$. These kind of equations arise in different fields of applied mathematics, such as lubrication problems for spreading droplets or thin films (for an excellent overview see [6] and the references therein) and also in modern quantum semiconductor design [25]. Due to their higher order nature and the lack of a suitable maximum principle, they pose challenging analytical and numerical problems. Even if strictly positive continuous solutions are available, standard discretizations often fail to feature this property. Nevertheless, the proof of positivity for the relevant discrete physical variables is of immense importance concerning the performance of algorithms and a posteriori interpretation of numerical solutions. In the existing literature the special structure of the equations is the key to the derivation of such uniform bounds and strongly depends on the degeneracy of the nonlinearity in the equations under consideration $[5,7,8,11,19]$.

In this paper we focus on a nonlinear fourth order parabolic system arising in quantum semiconductor modelling. The derivation and numerical treatment of new macroscopic quantum models is essential for electrical engineers to keep pace with the accelerating miniaturization in device fabrication. Reaching the decanano length scale quantum effects are no more negligible and the widely used classical semiconductor models become unapplicable. To overcome this problem much effort has been spent to incorporate the relevant quantum mechanical phenomena in the well understood classical models. The state of the art in quantum semiconductor device modelling ranges from microscopic models such as Schrödinger-Poisson systems [22] to macroscopic equations such as the quantum hydrodynamic model (QHD) [12-14]. During the last years a whole hierarchy

Keywords and phrases. Higher order parabolic PDE, positivity, semidiscretization, stability, convergence, semiconductors.

1 Fachbereich Mathematik, Universität Konstanz, 78457 Konstanz, Germany. e-mail: juengel@mathematik.uni-konstanz.de

2 Fachbereich Mathematik, Technische Universität Darmstadt, 64289 Darmstadt, Germany.

e-mail: pinnau@mathematik.tu-darmstadt.de 
of macroscopic models has been derived. They deal with macroscopic, fluid-type unknowns which allow for a natural interpretation of boundary conditions [24]. The models consist of balance equations for the particle density, current density and energy density and can be derived via a moment expansion from a many particle Schrödinger-Poisson system [15,18].

Most analytical and numerical work on these models was spent on the stationary equations, since the main interest was focused on the stationary current-voltage characteristics. Particularly for stationary simulations, a first moment version of the isothermal QHD, the quantum drift diffusion model (QDD) [2,4], proved to be quite promising since it allows a very efficient numerical treatment [27]. Only recently some results on the transient equations are available. The transient quantum drift diffusion model can be derived as a zero relaxation time limit in the rescaled QHD, which reads

$$
\begin{gathered}
n_{t}+\operatorname{div} J=0, \\
\tau_{\text {relax }}^{2} J_{t}+\tau_{\text {relax }}^{2} \operatorname{div}\left(\frac{J \otimes J}{n}\right)+\theta \nabla n+n \nabla V-\varepsilon^{2} n \nabla\left(\frac{\Delta \sqrt{n}}{\sqrt{n}}\right)=-J, \\
-\lambda^{2} \Delta V=n-C_{\text {dot }} .
\end{gathered}
$$

Here, the parameters are the scaled Planck constant $\varepsilon$, the scaled Debye length $\lambda$, the scaled temperature $\theta$ and the scaled relaxation time $\tau_{\text {relax }}$. The distribution of charged background ions is described by the doping profile $C_{\text {dot }}(x)$, which is assumed to be independent of time (for details see [25]). The variables are the electron density $n(x, t)$, the current density $J(x, t)$ and the electrostatic potential $V(x, t)$.

Note that the QHD is a dispersive regularization of the classical hydrodynamic model [17] which is of hyperbolic type. There are only few results available on the QHD and also its numerical treatment poses severe problems due to the presence of high frequency oscillations, comparable to the effects which can be observed in the context of the KdV equation [23]. Up to now it is not clear if the QHD generally allows for non-negative electron densities. The main difficulty of the analytical treatment of the QHD is the nonlinear third-order term. Fortunately, things improve significantly in the diffusive regime, i.e. for small relaxation times $\tau_{\text {relax }}$. A numerical study of the limit $\tau_{\text {relax }} \rightarrow 0$ for the stationary QHD can be found in [26], yielding a parabolic equation of fourth order.

The limiting transient system $\left(\tau_{\text {relax }}=0\right)$, stated on a bounded domain $\Omega$, can be written as

$$
\begin{gathered}
n_{t}=-\frac{\varepsilon^{2}}{2} \Delta^{2} n+\frac{\varepsilon^{2}}{2} \sum_{i, j=1}^{d} \partial_{x_{i}} \partial_{x_{j}}\left(\frac{\partial_{x_{i}} n \partial_{x_{j}} n}{n}\right)+\theta \Delta n+\operatorname{div}(n \nabla V) \\
-\lambda^{2} \Delta V=n-C_{\text {dot }}
\end{gathered}
$$

This is a fourth order nonlinear parabolic equation for the electron density $n$, which is self-consistently coupled to Poisson's equation for the potential $V$. In the case of vanishing quantum effects $(\varepsilon=0)$ this system reduces to the classical unipolar drift-diffusion model [21].

To get a well posed problem, system (1.1) has to be supplemented with appropriate boundary conditions. We assume that the boundary $\partial \Omega$ of the domain $\Omega$ splits into two disjoint parts $\Gamma_{D}$ and $\Gamma_{N}$, where $\Gamma_{D}$ models the Ohmic contacts of the device and $\Gamma_{N}$ represents the insulating parts of the boundary. Let $\nu$ denote the unit outward normal vector along $\partial \Omega$. The electron density is assumed to fulfil local charge neutrality at the Ohmic contacts:

$$
n=C_{\mathrm{dot}} \quad \text { on } \Gamma_{D}
$$

Concerning the potential we assume that it is a superposition of its equilibrium value and an applied biasing voltage $U$ at the Ohmic contacts, and that the electric field vanishes along the Neumann part of the boundary:

$$
V=V_{\text {eq }}+U \quad \text { on } \Gamma_{D}, \quad \nabla V \cdot \nu=0 \quad \text { on } \Gamma_{N}
$$


Further, it is natural to assume that there is no normal component of the current along the insulating part of the boundary and additionally, the normal component of the quantum current has to vanish:

$$
J \cdot \nu=0, \quad \nabla\left(\frac{\Delta \sqrt{n}}{\sqrt{n}}\right) \cdot \nu=0 \quad \text { on } \Gamma_{N} .
$$

Lastly, we require that no quantum effects occur at the contacts:

$$
\Delta \sqrt{n}=0 \quad \text { on } \Gamma_{D}
$$

These boundary conditions are physically motivated and commonly employed in quantum semiconductor modelling. The numerical investigations in [24] underline the reasonability of this choice.

System (1.4) is supplemented by the initial condition

$$
n(x, 0)=n_{0}(x) \quad \text { in } \Omega .
$$

Let us collect some results available for system (1.1). In [25] the dynamic stability of stationary states with a slightly different set of boundary conditions was established, at least for small scaled Planck constants and small applied biasing voltages. So far, there are only a few results available concerning the solvability of (1.1) due to the lack of an appropriate maximum principle ensuring the positivity of the electron density $n$. For zero temperature $(\theta=0)$ and vanishing electric field (1.1) simplifies to

$$
n_{t}=-\frac{\varepsilon^{2}}{2} \Delta^{2} n+\frac{\varepsilon^{2}}{2} \sum_{i, j=1}^{d} \partial_{x_{i}} \partial_{x_{j}}\left(\frac{\partial_{x_{i}} n \partial_{x_{j}} n}{n}\right) .
$$

Surprisingly, this equation also arises as a scaling limit in the study of interface fluctuations in a certain spin system. Bleher et al. [9] showed that there exists a unique positive classical solution locally in time in one space dimension, assuming strictly positive $H^{1}(\Omega)$-data and periodic boundary conditions. Their argument is based on the regularity of the semigroup generated by the Bilaplacian. The authors proved in [19] under much weaker assumptions the existence of a non-negative global solution $n$ in one space dimension, using an exponential transformation of variables which exploits the special nonlinear structure of equation (1.2).

Note that the leading order term in (1.2) is not degenerate, such that the well-known methods for lubricationtype equations

$$
h_{t}+\operatorname{div}(f(h) \nabla \Delta h)=0
$$

are unapplicable. During the last years the dynamics of equations of this type were thoroughly investigated using nonlinear entropy methods $[5,7,11]$. Numerically, there are several ways of dealing with equation (1.3). For instance, Bertozzi et al. [8] designed a space discretization using finite differences, which exhibits the same properties as the continuous equation. Barrett et al. [3] proposed a non-negativity preserving finite element method, where the non-negativity property is imposed as a constraint such that at each time level a variational inequality has to be solved. Recently, Grün et al. developed a finite element method without such a constraint [16]. All of them heavily rely on the nonlinear entropy estimates available due to the degeneracy and cannot be used for equation (1.2).

Concerning system (1.1) in one space dimension, a different numerical scheme was introduced by the authors, which proved to be stable and convergent [20]: writing equation (1.1a) in conservation form

$$
n_{t}=\operatorname{div}\left(n \nabla\left(-\varepsilon^{2} \frac{\Delta \sqrt{n}}{\sqrt{n}}+\theta \log (n)+V\right)\right)
$$

and introducing the quantum quasi Fermi level

$$
F=-\varepsilon^{2} \frac{\Delta \sqrt{n}}{\sqrt{n}}+\theta \log (n)+V
$$


yields the system

$$
\begin{gathered}
n_{t}=\operatorname{div}(n \nabla F), \\
-\varepsilon^{2} \frac{\Delta \sqrt{n}}{\sqrt{n}}+\theta \log (n)+V=F, \\
-\lambda^{2} \Delta V=n-C_{\mathrm{dot}} .
\end{gathered}
$$

Here, $-\varepsilon^{2} \Delta \sqrt{n} / \sqrt{n}$ is the so-called quantum Bohm potential. The additional boundary conditions

$$
F=U \quad \text { on } \Gamma_{D}, \quad \nabla F \cdot \nu=0 \quad \text { on } \Gamma_{N}
$$

are consistent with (1.1c)-(1.1f).

Then, an implicit time discretization by a backward Euler scheme for system (1.4) is suggested. The resulting sequence of elliptic systems proves to be uniquely solvable at each time step and moreover the semidiscrete solution is strictly positive. However, the positivity property relaxes in the limit to non-negativity. Due to the possible presence of vacuum $(n=0)$ it was not possible to identify the limiting quantum quasi Fermi level $F$ and to derive adequate bounds to establish convergence in several space dimensions.

In this paper we generalize this convergence result to the multi-dimensional case. From Remark 2.3 in [20] we learn that even for several space dimensions the semidiscretization possesses a strictly positive solution $n\left(x, t_{k}\right)$ as long as the lattice temperature $\theta$ is sufficiently large. Since there is no uniform lower bound on the electron density available we will assume this property and some regularity of the continuous solution. The positivity assumption seems reasonable due to the suggestive form of the nonlinearity and can also be proved for small data [9]. This regularity assumption has the benefit that we cannot only prove the desired convergence result but get also estimates on the rate of convergence which proves to be optimal for the Euler scheme.

The proof is based on a stability estimate which is a consequence of the boundedness of the entropy (or free energy)

$$
S(t)=\varepsilon^{2} \int_{\Omega}|\nabla \sqrt{n(t)}|^{2} \mathrm{~d} x+\theta \int_{\Omega} H(n(t)) \mathrm{d} x+\frac{\lambda^{2}}{2} \int_{\Omega}|\nabla V(t)|^{2} \mathrm{~d} x .
$$

In fact, $S$ is non-increasing in time (see [20]). Here, $H(s) \stackrel{\text { def }}{=} s(\log (s)-1)+1$ denotes a primitive of the logarithm.

The paper is organized as follows. In Section 2 we introduce the semidiscretization of (1.4). Section 3 is devoted to the proof of convergence in the multi-dimensional case, which relies on an energy estimate for the discrete solution. Imposing some natural assumptions we show that the scheme is convergent with the optimal order in some suitable norm.

\section{SEMidiscRETIZATION}

In this section we derive the implicit semidiscretization of (1.4) and state an existence and stability result for the discretized system at each time level. In particular, the positivity of the electron density is guaranteed.

For the following investigations we introduce the new variable $\rho=\sqrt{n}$. Then (1.4) reads:

$$
\begin{gathered}
\left(\rho^{2}\right)_{t}=\operatorname{div}\left(\rho^{2} \nabla F\right), \\
-\varepsilon^{2} \frac{\Delta \rho}{\rho}+\theta \log \left(\rho^{2}\right)+V=F, \\
-\lambda^{2} \Delta V=\rho^{2}-C_{\mathrm{dot}} .
\end{gathered}
$$

For the numerical treatment of (2.1) we employ a vertical line method and replace the transient problem by a sequence of elliptic problems. 
Let $T>0$ be given. We divide the time interval $[0, T]$ into $N$ subintervals by introducing the temporal mesh $\left\{t_{k}: k=0, \ldots, N\right\}$, where $0=t_{0}<t_{1}<\ldots<t_{N}=T$. We set $\tau_{k} \stackrel{\text { def }}{=} t_{k}-t_{k-1}$ and define the maximal subinterval length $\tau \stackrel{\text { def }}{=} \max _{k=1, \ldots, N} \tau_{k}$. We assume that the partition fulfils

$$
\tau \rightarrow 0 \quad \text { as } N \rightarrow \infty
$$

For any Banach space $B$ we define

$$
P C_{N}(0, T ; B) \stackrel{\text { def }}{=}\left\{v^{\tau}:(0, T] \rightarrow B:\left.v^{\tau}\right|_{\left(t_{k-1}, t_{k}\right]} \equiv \text { const. for } k=1, \ldots, N\right\}
$$

and introduce the abbreviation $v_{k}=v^{\tau}(t)$ for $t \in\left(t_{k-1}, t_{k}\right]$ and $k=1, \ldots, N$. Further, let $\tilde{v}^{\tau}$ denote the linear interpolant of $v^{\tau} \in P C_{N}\left(0, T ; L^{2}(\Omega)\right)$ given by

$$
\tilde{v}^{\tau}(t, x)=\frac{t-t_{k-1}}{\tau_{k}}\left(v_{k}-v_{k-1}\right)+v_{k-1}, \quad \text { for } x \in \Omega, \quad t \in\left(t_{k-1}, t_{k}\right]
$$

Now we discretize (2.1) using an implicit Euler scheme:

Set $\rho_{0}=\sqrt{n(0)}$. For $k=1, \ldots, N$ solve recursively the elliptic systems

$$
\begin{gathered}
\frac{1}{\tau_{k}}\left(\rho_{k}^{2}-\rho_{k-1}^{2}\right)=\operatorname{div}\left(\rho_{k}^{2} \nabla F_{k}\right), \\
-\varepsilon^{2} \frac{\Delta \rho_{k}}{\rho_{k}}+\theta \log \left(\rho_{k}^{2}\right)+V_{k}=F_{k} \\
-\lambda^{2} \Delta V_{k}=\rho_{k}^{2}-C_{\mathrm{dot}}
\end{gathered}
$$

subject to the boundary conditions

$$
\begin{array}{ll}
\rho_{k}=\rho_{D}, \quad F_{k}=F_{D}, \quad V_{k}=V_{D} & \text { on } \Gamma_{D}, \\
\nabla \rho_{k} \cdot \nu=\nabla F_{k} \cdot \nu=\nabla V_{k} \cdot \nu=0 & \text { on } \Gamma_{N},
\end{array}
$$

where

$$
\rho_{D}=\sqrt{C_{\mathrm{dot}}}, \quad F_{D}=U, \quad V_{D}=-\theta \log \left(C_{\mathrm{dot}}\right)+U .
$$

Then the approximate solution to $(2.1)$ is given by $\left(\rho^{\tau}, F^{\tau}, V^{\tau}\right)$.

We use the standard notation for Sobolev spaces (see [1]), denoting the norm of $W^{m, p}(\Omega)\left(m \in \mathbb{R}_{0}^{+}, p \in[1, \infty]\right)$ by $\|\cdot\|_{W^{m, p}(\Omega)}$. In the special case $p=2$ we use $H^{m}(\Omega)$ instead of $W^{m, 2}(\Omega)$. Further, let $H_{0}^{m}(\Omega)$ be the closure of $C_{c}^{\infty}(\Omega)$ with respect to the $H^{m}(\Omega)$ norm and let $H_{0}^{1}\left(\Omega \cup \Gamma_{N}\right)$ for $\Gamma_{N} \subset \partial \Omega$ be the closure of $C_{c}^{\infty}\left(\Omega \cup \Gamma_{N}\right)$ with respect to the $H^{1}(\Omega)$ norm [29]. Moreover, for any Banach space $B$ we define the space $L^{p}(0, T ; B)$ with $p \in[1, \infty]$ consisting of all measurable functions $\varphi:(0, T) \rightarrow B$ for which the norm

$$
\begin{aligned}
& \|\varphi\|_{L^{p}(0, T ; B)} \stackrel{\text { def }}{=}\left(\int_{0}^{T}\|\varphi\|_{B}^{p} \mathrm{~d} t\right)^{1 / p}, \quad p \in[1, \infty), \\
& \|\varphi\|_{L^{\infty}(0, T ; B)} \stackrel{\text { def }}{=} \sup _{t \in(0, T)}\|\varphi(t)\|_{B}, \quad p=\infty,
\end{aligned}
$$

is finite. If the time interval is clear we shortly write $\|\cdot\|_{L^{p}(B)}$.

Naturally, we have to assume some regularity properties on the data. For the subsequent considerations we impose the following assumptions: 
A.1 Let $\Omega \subset \mathbb{R}^{d}, d=1,2$ or 3 , be a bounded domain with boundary $\partial \Omega \in C^{1,1}$. The boundary $\partial \Omega$ is piecewise regular and splits into two disjoint parts $\Gamma_{N}$ and $\Gamma_{D}$. The set $\Gamma_{D}$ has nonvanishing $(d-1)$ dimensional Lebesgue-measure. $\Gamma_{N}$ is closed.

A.2 The boundary data fulfils (2.4) and

$$
\begin{gathered}
\rho_{D} \in H^{4}(\Omega), \quad \inf _{\Omega} \rho_{D}>0, \quad \nabla \rho_{D} \cdot \nu=0 \text { on } \Gamma_{N}, \\
F_{D} \in C^{2, \gamma}(\bar{\Omega}) \quad \text { for } \gamma \in\left(0, \frac{1}{2}\right), \quad F_{D} \leq-\bar{F}_{D}<0, \\
V_{D} \in C^{2, \gamma}(\bar{\Omega}),
\end{gathered}
$$

and the initial datum satisfies $\rho_{0} \in H^{2}(\Omega)$. Further, $C_{\text {dot }} \in C^{0, \gamma}(\bar{\Omega})$.

A.3 Let $\gamma \in(0,1)$ and $a \in C^{0, \gamma}(\bar{\Omega})$ with $a \geq \underline{a}>0$. Then there exists a constant $K=K\left(\Omega, \Gamma_{D}, \Gamma_{N}, a, d, \gamma\right)>$ 0 such that for $f \in C^{0, \gamma}(\bar{\Omega})$ and $u_{D} \in C^{2, \gamma}(\bar{\Omega})$ there exists a solution $u \in C^{2, \gamma}(\bar{\Omega})$ of

$$
\operatorname{div}(a \nabla u)=f, \quad u-u_{D} \in H_{0}^{1}\left(\Omega \cup \Gamma_{N}\right),
$$

which fulfils

$$
\|u\|_{C^{2, \gamma}(\bar{\Omega})} \leq K\left(\left\|u_{D}\right\|_{C^{2, \gamma}(\bar{\Omega})}+\|f\|_{C^{0, \gamma}(\bar{\Omega})}\right)
$$

\section{Remark 2.1.}

(a) Assumption A.3 is essentially a restriction on the geometry of $\Omega$. It is fulfilled in the case where the Dirichlet and the Neumann boundary do not meet, i.e. $\bar{\Gamma}_{D} \cap \Gamma_{N}=\emptyset[29]$.

(b) The restriction $F_{D} \leq-\bar{F}_{D}$ on the quantum quasi Fermi level is purely technical. From the physical point of view the device behaviour is independent of a shift $F \mapsto F+\alpha, V \mapsto V+\alpha, \alpha \in \mathbb{R}$.

(c) For a smoother presentation we assume that the boundary conditions are independent of time.

In [20] an existence theorem for (2.3) is proved, which reads in the multi-dimensional case:

Proposition 2.2 (Assume A.1-A.3). Furthermore, let $k \in\{1, \ldots, N\}$ and let $\rho_{k-1} \in C^{0, \gamma}(\bar{\Omega})$. Then there exists a constant $\theta_{0}>0$ such that for all $\theta>\theta_{0}$ system $(2.3)$ possesses a solution $\left(\rho_{k}, F_{k}, V_{k}\right)$, fulfilling

(a) $\left(\rho_{k}, F_{k}, V_{k}\right) \in H^{2}(\Omega) \times C^{2, \gamma}(\bar{\Omega}) \times C^{2, \gamma}(\bar{\Omega})$ for $0<\gamma<\frac{1}{2}$,

(b) $\exists c_{k}>0: \quad \rho_{k} \geq c_{k}>0$ in $\Omega$.

Furthermore, the approximate solution is stable in the following sense (see [20], Cor. 2.5).

Lemma 2.3 (Assume A.1-A.3). For $k=1, \ldots, N$ let $\left(\rho_{k}, F_{k}, V_{k}\right)$ be the recursively defined solution of (2.3) and $\left(\rho^{\tau}, F^{\tau}, V^{\tau}\right) \in P C_{N}\left(0, T ; H^{2}(\Omega) \times C^{2, \gamma}(\bar{\Omega}) \times C^{2, \gamma}(\bar{\Omega})\right)$. Then $\rho^{\tau} \in L^{\infty}\left(0, T ; H^{1}(\Omega)\right)$ and $\rho^{\tau} \nabla F^{\tau} \in L^{2}(0, T$; $\left.L^{2}(\Omega)\right)$. Further, there exists a positive constant $c$, independent of $\tau$, such that

$$
\left\|\rho^{\tau}\right\|_{L^{\infty}\left(H^{1}\right)}+\left\|V^{\tau}\right\|_{L^{\infty}\left(H^{1}\right)}+\left\|\rho^{\tau} \nabla F^{\tau}\right\|_{L^{2}\left(L^{2}\right)} \leq c .
$$

Remark 2.4. In the one-dimensional case it is possible to prove (see [20], Th. 3.3) the existence of a subsequence, again denoted by $\left(\rho^{\tau}, F^{\tau}, V^{\tau}\right)$, such that

$$
\begin{aligned}
\rho^{\tau} \rightarrow \rho & \text { weakly in } L^{2}\left(0, T ; H^{2}(\Omega)\right), \\
\rho^{\tau} \rightarrow \rho & \text { strongly in } C^{0}\left([0, T] ; C^{0, \gamma}(\bar{\Omega})\right), \\
\left(\rho^{\tau}\right)^{2} F_{x}^{\tau} \rightarrow J & \text { weakly in } L^{2}\left(0, T ; L^{2}(\Omega)\right), \\
V^{\tau} \rightarrow V & \text { strongly in } C^{0}\left([0, T] ; C^{2, \gamma}(\bar{\Omega})\right),
\end{aligned}
$$

as $\tau \rightarrow 0$, where $(\rho, J, V)$ is a weak solution of the continuous problem (2.1). Note, that due to possible presence of vacuum it is not possible to identify the limiting quantum quasi Fermi level $F$. 


\section{Convergence in Several Space Dimension}

In this section we prove the convergence of the numerical scheme given by (2.3) in the multi-dimensional case. Here, the a priori bounds on the approximate solution in Lemma 2.3 are not sufficient to guarantee convergence, since the argument depends strongly on an $L^{\infty}\left(0, T ; L^{\infty}(\Omega)\right)$-bound on $\rho^{\tau}$ (see [20]). In one space dimension this is an immediate consequence of the estimate $(2.5)$ and the embedding $H^{1}(\Omega) \hookrightarrow L^{\infty}(\Omega)$. Further, the identification of the quantum quasi Fermi level $F$ is only possible for a non-vanishing electron density $n$, which is also essential for the derivation of the convergence result, since it allows to prove the standard regularity $\rho^{\tau} \in L^{2}\left(0, T ; H^{2}(\Omega)\right)$ for fourth order parabolic equations. Clearly, the derivation of convergence rates also requires some regularity with respect to time. Here, we assume $\rho_{t t} \in L^{2}\left(0, T ; L^{2}(\Omega)\right)$, which is already employed for the numerical analysis of the second order parabolic classical drift diffusion system. In fact, no analytical results on system (1.4) are available in several space dimensions. Thus, we have to state additional assumptions on the sequence of approximating solutions. These enable us to give even error estimates, which exhibit the optimal order of convergence for the implicit Euler scheme.

Theorem 3.1 (Assume A.1-A.3). For $k=1, \ldots, N$ let $\left(\rho_{k}, F_{k}, V_{k}\right)$ be the recursively defined solution of (2.3) and $\left(\rho^{\tau}, F^{\tau}, V^{\tau}\right) \in P C_{N}\left(0, T ; H^{2}(\Omega) \times C^{2, \gamma}(\bar{\Omega}) \times C^{2, \gamma}(\bar{\Omega})\right)$. Assuming

$$
\text { A.4 } \exists \delta \in(0,1) \quad \forall \tau>0: \quad \delta \leq \rho^{\tau} \leq \delta^{-1}, \quad\left\|\rho^{\tau}\right\|_{L^{\infty}\left(0, T ; H^{2}(\Omega)\right)} \leq \delta^{-1} ;
$$

there exists a subsequence, again denoted by $\left(\rho^{\tau}, F^{\tau}, V^{\tau}\right)$, such that

$$
\begin{array}{cl}
\rho^{\tau} \rightarrow \rho & \text { weakly in } L^{2}\left(0, T ; H^{2}(\Omega)\right), \\
\rho^{\tau} \rightarrow \rho & \text { strongly in } C^{0}\left([0, T] ; C^{0, \gamma}(\bar{\Omega})\right), \\
F^{\tau} \rightarrow F & \text { strongly in } C^{0}\left([0, T] ; H^{1}(\Omega)\right), \\
V^{\tau} \rightarrow V & \text { strongly in } C^{0}\left([0, T] ; C^{2, \gamma}(\bar{\Omega})\right),
\end{array}
$$

as $\tau \rightarrow 0$, where $(\rho, F, V)$ is a solution of the continuous problem (2.1).

Furthermore, if the embedding $H^{2}(\Omega) \hookrightarrow W^{m, p}(\Omega)$ is continuous for some $m \geq 0, p \geq 1$ and

A.5 $\rho \in H^{2}\left(0, T ; L^{2}(\Omega)\right)$;

then there exists a constant $\tau_{0}=\tau_{0}(\Omega, \lambda, \delta)>0$ such that for $\tau \in\left[0, \tau_{0}\right)$ we have the following error estimate

$$
\left\|\rho^{\tau}-\rho\right\|_{L^{\infty}\left(L^{2}\right)}+\varepsilon^{2}\left\|\rho^{\tau}-\rho\right\|_{L^{2}\left(W^{m, p}\right)}+\left\|F^{\tau}-F\right\|_{L^{\infty}\left(H^{2}\right)}+\left\|V^{\tau}-V\right\|_{L^{\infty}\left(H^{2}\right)} \leq C \mathrm{e}^{\alpha T} \tau,
$$

for some positive constants $\alpha=\alpha\left(\Omega, \lambda, \delta, \tau_{0}\right)$ and $C=C\left(\Omega, \lambda, \delta, \tau_{0}\right)$.

\section{Remark 3.2.}

(a) Assumption A.4 allows us to verify the strong convergence $F^{\tau} \rightarrow F$ which yields the identification $J=\rho^{2} \nabla F$ for the limiting current density. Notice that this extends the one-dimensional result (see Rem. 2.4).

(b) Already in the classical regime $(\varepsilon=0)$ assumption $\mathbf{A . 5}$ is employed for the derivation of the optimal order of convergence in one space dimension [10]. Remarkably, it is also sufficient in this higher order system in several space dimensions.

(c) An inspection of the proof of Theorem 3.1 shows that the last assumption in $\mathbf{A . 4}$ can be replaced by the weaker condition $\left\|\rho^{\tau}\right\|_{L^{\infty}(0, T ; Z)} \leq \delta^{-1}$, and $Z$ is a Sobolev space which embeddes compactly into $W^{1,3}(\Omega)$.

For the convergence result we also need some bound in the energy norm and on the time derivative. To this purpose we introduce the linear interpolant of $\left(\rho^{\tau}\right)^{2} \in P C_{N}\left(0, T ; L^{2}(\Omega)\right)$, defined by

$$
\tilde{n}^{\tau}(t, x) \stackrel{\text { def }}{=} \frac{t-t_{k}}{\tau_{k}}\left(\rho_{k}^{2}(x)-\rho_{k-1}^{2}(x)\right)+\rho_{k-1}^{2}(x), \quad x \in \Omega, \quad t \in\left(t_{k-1}, t_{k}\right] .
$$


Following the outlines of the proof of Lemma 3.1 and Lemma 3.2 in [20] one verifies that A.4 is sufficient to derive the following additional stability estimates.

Lemma 3.3 (Assume A.1-A.4). For $k=1, \ldots, N$ let $\left(\rho_{k}, F_{k}, V_{k}\right)$ be the recursively defined solution of (2.3) and $\left(\rho^{\tau}, F^{\tau}, V^{\tau}\right) \in P C_{N}\left(0, T ; H^{2}(\Omega) \times C^{2, \gamma}(\bar{\Omega}) \times C^{2, \gamma}(\bar{\Omega})\right)$. Then $\rho^{\tau} \in L^{2}\left(0, T ; H^{2}(\Omega)\right)$ and $\tilde{n}^{\tau} \in H^{1}(0, T$; $\left.H^{-1}(\Omega)\right)$. Further, there exists a positive constant $c$, independent of $\tau$, such that

$$
\left\|\rho^{\tau}\right\|_{L^{2}\left(H^{2}\right)} \leq c \quad \text { and } \quad\left\|\tilde{n}_{t}^{\tau}\right\|_{L^{2}\left(H^{-1}\right)} \leq c .
$$

For the proof of Theorem 3.1 we need the monotonicity of the quantum "operator"

$$
A(\rho)=\frac{1}{\rho} \operatorname{div}\left(\rho^{2} \nabla \frac{\Delta \rho}{\rho}\right), \quad \rho \in H^{4}(\Omega) .
$$

Lemma 3.4 (Assume A.1 and A.3). Choose $m \geq 0, p \geq 1$ such that the continuous embedding $H^{2}(\Omega) \hookrightarrow$ $W^{m, p}(\Omega)$ holds. Then there exists for all $\beta \in \mathbb{R}$ and all $\delta \in(0,1)$ a constant $M=M(\Omega, \beta, \delta)>0$ such that for all $\rho \in H^{2}(\Omega)$ with $\delta \leq \rho \leq 1 / \delta$ and all $\phi \in H^{2}(\Omega) \cap H_{0}^{1}\left(\Omega \cup \Gamma_{N}\right)$ it holds

$$
\int_{\Omega} \rho^{\beta}\left|\operatorname{div}\left(\rho^{2} \nabla\left(\frac{\phi}{\rho}\right)\right)\right|^{2} \mathrm{~d} x \geq M\|\phi\|_{W^{m, p}(\Omega)}^{2} .
$$

The proof of Lemma 3.4 is a slight generalization of the one in [25] (Th. 3.7). It follows the monotonicity result.

Lemma 3.5 (Assume A.1). Let $u, v \in H^{4}(\Omega)$ be such that $u, v \geq \delta>0$ in $\Omega$ and

$$
\begin{aligned}
u-v=0, \quad \Delta u=\Delta v=0 & \text { on } \Gamma_{D}, \\
\nabla(u-v) \cdot \nu=0, \quad \nabla \frac{\Delta u}{u} \cdot \nu=\nabla \frac{\Delta v}{v} \cdot \nu=0 & \text { on } \Gamma_{N} .
\end{aligned}
$$

Then

$$
\int_{\Omega}(A(u)-A(v))(u-v) \mathrm{d} x=\int_{\Omega} \frac{1}{u v}\left|\operatorname{div}\left(u^{2} \nabla \frac{u-v}{u}\right)\right|^{2} \mathrm{~d} x .
$$

Proof. By integration by parts we obtain

$$
\begin{aligned}
\int_{\Omega}(A(u)-A(v))(u-v) \mathrm{d} x & =\int_{\Omega}\left(\frac{\Delta u}{u} \operatorname{div}\left(u^{2} \nabla \frac{u-v}{u}\right)-\frac{\Delta v}{v} \operatorname{div}\left(v^{2} \nabla \frac{u-v}{v}\right)\right) \mathrm{d} x \\
& =\int_{\Omega}\left(\frac{v \Delta u-u \Delta v}{u v} \operatorname{div}\left(u^{2} \nabla \frac{u-v}{u}\right)-\frac{\Delta v}{v} \operatorname{div}\left(v^{2} \nabla \frac{u-v}{v}-u^{2} \nabla \frac{u-v}{u}\right)\right) \mathrm{d} x .
\end{aligned}
$$

Since

this implies

$$
v^{2} \nabla \frac{u-v}{v}-u^{2} \nabla \frac{u-v}{u}=0 \quad \text { in } \Omega
$$

$$
\int_{\Omega}(A(u)-A(v))(u-v) \mathrm{d} x=\int_{\Omega} \frac{1}{u v}\left|\operatorname{div}\left(u^{2} \nabla \frac{u-v}{u}\right)\right|^{2} \mathrm{~d} x .
$$

Now we are in the position to prove Theorem 3.1. The first part of proof is a slight generalization of the one for Theorem 3.3 in [20]. However, we include it for the sake of a completeness.

Proof of Theorem 3.1. We choose a sequence of partitions of $[0, T]$ satisfying (2.2). According to Lemma 3.3 $\left(\rho^{\tau}\right)$ is bounded in $L^{2}\left(0, T ; H^{2}(\Omega)\right)$. We may choose a subsequence, again denoted by $\left(\rho^{\tau}\right)$, such that, as $\tau \rightarrow 0$,

$$
\rho^{\tau} \rightarrow \rho \quad \text { weakly in } L^{2}\left(0, T, H^{2}(\Omega)\right) .
$$


Further, we have due to Lemma 3.3 and Lemma 2.3 that $\tilde{n}^{\tau} \in H^{1}\left(0, T ; H^{-1}(\Omega)\right)$. Since the embedding $H^{2}(\Omega) \hookrightarrow C^{0, \gamma}(\bar{\Omega})$ is compact for $1 \leq d \leq 3$ and $0<\gamma<1 / 2$ we deduce from Aubin's lemma [28] that

$$
L^{\infty}\left(0, T ; H^{2}(\Omega)\right) \cap H^{1}\left(0, T ; H^{-1}(\Omega)\right) \hookrightarrow C^{0}\left(0, T ; C^{0, \gamma}(\bar{\Omega})\right) \quad \text { compactly. }
$$

Hence, using assumption A.4, there exists a subsequence, not relabeled, such that

$$
\tilde{n}^{\tau} \rightarrow n \quad \text { strongly in } C^{0}\left(0, T ; C^{0, \gamma}(\bar{\Omega})\right) .
$$

The reader easily verifies the identification $n=\rho^{2}$. By assumption A.4 and inequality (2.5), we get a uniform estimate for $\tilde{\rho}_{t}^{\tau}$ in $L^{2}\left(0, T ; H^{-1}(\Omega)\right)$. Hence, the compact embedding

$$
L^{2}\left(0, T ; H^{2}(\Omega)\right) \cap H^{1}\left(0, T ; H^{-1}(\Omega)\right) \hookrightarrow L^{2}\left(0, T ; H^{1}(\Omega)\right)
$$

implies that (up to a subsequence)

$$
\tilde{\rho}^{\tau} \rightarrow \rho \quad \text { strongly in } L^{2}\left(0, T ; H^{1}(\Omega)\right)
$$

and consequently,

$$
\rho^{\tau} \rightarrow \rho \quad \text { strongly in } L^{2}\left(0, T ; H^{1}(\Omega)\right) .
$$

Standard results from elliptic theory and A.2 imply now

$$
V^{\tau} \rightarrow V \quad \text { strongly in } C^{0}\left(0, T, C^{2, \gamma}(\bar{\Omega})\right) .
$$

Defining $J^{\tau}=\left(\rho^{\tau}\right)^{2} \nabla F^{\tau}$ we deduce from Lemma 2.3 that $\left(J^{\tau}\right)$ is bounded in $L^{2}\left(0, T, L^{2}(\Omega)\right)$, such that

$$
J^{\tau} \rightarrow J \quad \text { weakly in } L^{2}\left(0, T ; L^{2}(\Omega)\right) .
$$

Now, the convergence of $\left(F^{\tau}\right)$ to $F$ follows from the uniform bound $\rho^{\tau} \geq \delta$ combined with standard elliptic theory. Further, $J=\rho^{2} \nabla F$.

The derived convergence properties are by far sufficient to pass to the limit in the weak formulation of (2.3).

In order to estimate the rate of convergence, we need some regularity properties for $\rho_{k}$ and $\rho\left(t_{k}\right)$. From

$$
-\varepsilon^{2} \Delta \rho_{k}=\rho_{k}\left(F_{k}-\theta \log \left(\rho_{k}^{2}\right)-V_{k}\right) \in H^{2}(\Omega)
$$

and assumption A.2 we obtain $\rho_{k} \in H^{4}(\Omega)$. The compact embedding

$$
L^{\infty}\left(0, T ; H^{2}(\Omega)\right) \cap H^{1}\left(0, T ; H^{-1}(\Omega)\right) \hookrightarrow C^{0}\left([0, T] ; C^{0, \gamma}(\bar{\Omega})\right)
$$

implies that $\rho$ is continuous in $C^{0}\left([0, T] ; C^{0, \gamma}(\bar{\Omega})\right)$ and hence,

$$
-\varepsilon^{2} \Delta \rho=\rho\left(F-\theta \log \left(\rho^{2}\right)-V\right) \in C^{0}\left([0, T] ; C^{0, \gamma}(\bar{\Omega})\right) .
$$

By a bootstrapping argument, it follows $\rho \in C^{0}\left([0, T] ; H^{4}(\Omega)\right)$.

Now let $k \in\{1, \ldots, N\}$ be fixed. We take the difference of

$$
2 \rho_{t}=\frac{1}{\rho} \operatorname{div}\left(\rho^{2} \nabla F\right)
$$

and

$$
\frac{2}{\tau_{k}}\left(\rho_{k}-\rho_{k-1}\right)-\frac{1}{\tau_{k}} \frac{\left(\rho_{k}-\rho_{k-1}\right)^{2}}{\rho_{k}}=\frac{1}{\rho_{k}} \operatorname{div}\left(\rho_{k}^{2} \nabla F_{k}\right) .
$$


Note that $\rho_{k}, \rho \geq \delta$. Further, by Taylor's expansion we have

$$
\rho\left(t_{k}\right)=\rho\left(t_{k-1}\right)+\rho_{t}\left(t_{k}\right) \tau_{k}+\frac{1}{2} \int_{t_{k-1}}^{t_{k}} \rho_{t t}(s)\left(s-t_{k-1}\right) \mathrm{d} s
$$

Setting

$$
f_{k} \stackrel{\text { def }}{=} \frac{1}{2} \int_{t_{k-1}}^{t_{k}} \rho_{t t}(s)\left(s-t_{k-1}\right) \mathrm{d} s
$$

and defining the error

$$
e_{k} \stackrel{\text { def }}{=} \rho_{k}-\rho\left(t_{k}\right)
$$

we finally end up with

$$
\frac{2}{\tau_{k}}\left(e_{k}-e_{k-1}\right)-\frac{1}{\tau_{k}} \frac{\left(\rho_{k}-\rho_{k-1}\right)^{2}}{\rho_{k}}+\frac{2}{\tau_{k}} f_{k}=\frac{1}{\rho_{k}} \operatorname{div}\left(\rho_{k}^{2} \nabla F_{k}\right)-\frac{1}{\rho\left(t_{k}\right)} \operatorname{div}\left(\rho\left(t_{k}\right)^{2} \nabla F\left(t_{k}\right)\right) .
$$

Now we use $\phi=\tau_{k} e_{k}$ as test function, which yields

$$
\begin{aligned}
2 \int_{\Omega}\left(e_{k}-e_{k-1}\right) e_{k} \mathrm{~d} x-\int_{\Omega} \frac{\left(\rho_{k}-\rho_{k-1}\right)^{2}}{\rho_{k}} e_{k} \mathrm{~d} x+2 \int_{\Omega} f_{k} e_{k} \mathrm{~d} x= \\
\tau_{k} \int_{\Omega}\left[\frac{1}{\rho_{k}} \operatorname{div}\left(\rho_{k}^{2} \nabla F_{k}\right)-\frac{1}{\rho\left(t_{k}\right)} \operatorname{div}\left(\rho\left(t_{k}\right)^{2} \nabla F\left(t_{k}\right)\right)\right] e_{k} \mathrm{~d} x .
\end{aligned}
$$

We estimate termwise starting on the left-hand side.

Using the identity $2 r(r-s)=r^{2}-s^{2}+(r-s)^{2}$ we get

$$
2 \int_{\Omega}\left(e_{k}-e_{k-1}\right) e_{k} \mathrm{~d} x=\left\|e_{k}\right\|_{L^{2}(\Omega)}^{2}-\left\|e_{k-1}\right\|_{L^{2}(\Omega)}^{2}+\left\|e_{k}-e_{k-1}\right\|_{L^{2}(\Omega)}^{2} .
$$

Let $\eta=\delta / \max _{k=1, \ldots, N}\left\|\rho_{k}\right\|_{L^{\infty}(\Omega)}=\delta^{2}$. It holds

$$
\begin{aligned}
-\int_{\Omega} \frac{\left(\rho_{k}-\rho_{k-1}\right)^{2}}{\rho_{k}} e_{k} \mathrm{~d} x & \geq-(1-\eta) \int_{\Omega}\left(\rho_{k}-\rho_{k-1}\right)^{2} \mathrm{~d} x \\
& =-(1-\eta) \int_{\Omega}\left(e_{k}-e_{k-1}+\rho\left(t_{k}\right)-\rho\left(t_{k-1}\right)\right)^{2} \mathrm{~d} x \\
& \geq-\left\|e_{k}-e_{k-1}\right\|_{L^{2}(\Omega)}^{2}-\frac{1-\eta}{\eta} \int_{\Omega}\left(\rho_{t}\left(t_{k}\right) \tau_{k}+f_{k}\right)^{2} \mathrm{~d} x
\end{aligned}
$$

where we used Taylor's expansion and Young's inequality. Trivially, it holds

$$
-2 \int_{\Omega} f_{k} e_{k} \mathrm{~d} x \leq 2\left\|f_{k}\right\|_{L^{2}(\Omega)}^{2}+\frac{1}{2}\left\|e_{k}\right\|_{L^{2}(\Omega)}^{2} .
$$


The right-hand side of (3.4) can be estimated using integration by parts.

$$
\begin{aligned}
& \tau_{k} \int_{\Omega}\left[\frac{1}{\rho_{k}} \operatorname{div}\left(\rho_{k}^{2} \nabla F_{k}\right)-\frac{1}{\rho\left(t_{k}\right)} \operatorname{div}\left(\rho\left(t_{k}\right)^{2} \nabla F\left(t_{k}\right)\right)\right] e_{k} \mathrm{~d} x \\
& =-\tau_{k} \varepsilon^{2} \int_{\Omega}\left(A\left(\rho_{k}\right)-A\left(\rho\left(t_{k}\right)\right)\right)\left(\rho_{k}-\rho\left(t_{k}\right)\right) \mathrm{d} x \\
& \quad+2 \tau_{k} \theta \int_{\Omega}\left[\Delta \rho_{k}+\frac{\left|\nabla \rho_{k}\right|^{2}}{\rho_{k}}-\Delta \rho\left(t_{k}\right)-\frac{\left|\nabla \rho\left(t_{k}\right)\right|^{2}}{\rho\left(t_{k}\right)}\right] e_{k} \mathrm{~d} x \\
& \quad+\tau_{k} \int_{\Omega}\left[2 \nabla \rho_{k} \nabla V_{k}-2 \nabla \rho\left(t_{k}\right) \nabla V\left(t_{k}\right)+\rho_{k} \Delta V_{k}-\rho\left(t_{k}\right) \Delta V\left(t_{k}\right)\right] e_{k} \mathrm{~d} x \\
& \leq-\tau_{k} \varepsilon^{2} \int_{\Omega}\left(A\left(\rho_{k}\right)-A\left(\rho\left(t_{k}\right)\right)\right)\left(\rho_{k}-\rho\left(t_{k}\right)\right) \mathrm{d} x \\
& \quad-2 \tau_{k} \theta \int_{\Omega}\left|\frac{\rho\left(t_{k}\right)}{\rho_{k}} \nabla \rho_{k}-\frac{\rho_{k}}{\rho\left(t_{k}\right)} \nabla \rho\left(t_{k}\right)\right|^{2} \mathrm{~d} x \\
& \quad+\tau_{k}\left[2 \nabla \rho_{\Omega} \nabla V_{k}-2 \nabla \rho\left(t_{k}\right) \nabla V\left(t_{k}\right)+\rho_{k} \Delta V_{k}-\rho\left(t_{k}\right) \Delta V\left(t_{k}\right)\right] e_{k} \mathrm{~d} x .
\end{aligned}
$$

The last term can be handled as follows:

$$
\begin{aligned}
& \tau_{k} \int_{\Omega}\left[2 \nabla \rho_{k} \nabla V_{k}-2 \nabla \rho\left(t_{k}\right) \nabla V\left(t_{k}\right)+\rho_{k} \Delta V_{k}-\rho\left(t_{k}\right) \Delta V\left(t_{k}\right)\right] e_{k} \mathrm{~d} x \\
& =\tau_{k} \int_{\Omega}\left[2 \nabla e_{k} \nabla V_{k}-2 \nabla \rho\left(t_{k}\right) \nabla\left(V\left(t_{k}\right)-V_{k}\right)+\rho_{k} \Delta V_{k}-\rho\left(t_{k}\right) \Delta V\left(t_{k}\right)\right] e_{k} \mathrm{~d} x \\
& =\tau_{k} \int_{\Omega}\left[-e_{k}^{2} \Delta V_{k}-2 \nabla \rho\left(t_{k}\right) \nabla\left(V\left(t_{k}\right)-V_{k}\right) e_{k}+e_{k}^{2} \Delta V_{k}-\rho\left(t_{k}\right) \Delta\left(V\left(t_{k}\right)-V_{k}\right) e_{k}\right] \mathrm{d} x \\
& =-2 \tau_{k} \int_{\Omega} \nabla \rho\left(t_{k}\right) \nabla\left(V\left(t_{k}\right)-V_{k}\right) e_{k} \mathrm{~d} x-\tau_{k} \int_{\Omega} \rho\left(t_{k}\right)\left(\rho\left(t_{k}\right)+\rho_{k}\right) e_{k}^{2} \mathrm{~d} x \\
& \leq-2 \tau_{k} \int_{\Omega} \nabla \rho\left(t_{k}\right) \nabla\left(V\left(t_{k}\right)-V_{k}\right) e_{k} \mathrm{~d} x \\
& \leq 2 \tau_{k}\left\|\nabla \rho\left(t_{k}\right)\right\|_{L^{3}(\Omega)}\left\|\nabla\left(V\left(t_{k}\right)-V_{k}\right)\right\|_{L^{6}(\Omega)}\left\|e_{k}\right\|_{L^{2}(\Omega)} .
\end{aligned}
$$

The compact embedding

$$
L^{\infty}\left(0, T ; H^{2}(\Omega)\right) \cap H^{1}\left(0, T ; H^{-1}(\Omega)\right) \hookrightarrow C^{0}\left([0, T] ; W^{1,3}(\Omega)\right)
$$

yields the uniform bound $\left\|\nabla \rho\left(t_{k}\right)\right\|_{L^{3}(\Omega)} \leq c_{0}$.

From the boundary conditions for $\rho_{k}, F_{k}$ and $V_{k}$ (see $(2.3 \mathrm{~d}),(2.3 \mathrm{e})$ and $\left.(2.4)\right)$ we conclude that

$$
\begin{aligned}
\nabla \rho_{k} \cdot \nu & =\nabla \frac{\Delta \rho_{k}}{\rho_{k}} \cdot \nu=0 \quad \text { in the sense of } L^{2}\left(\Gamma_{N}\right), \\
\rho_{k} & =\rho_{D}, \quad \Delta \rho_{k}=0 \quad \text { in the sense of } L^{2}\left(\Gamma_{D}\right) .
\end{aligned}
$$

Similarly,

$$
\begin{aligned}
\nabla \rho\left(t_{k}\right) \cdot \nu & =\nabla \frac{\Delta \rho\left(t_{k}\right)}{\rho\left(t_{k}\right)} \cdot \nu=0 \quad \text { in the sense of } L^{2}\left(\Gamma_{N}\right), \\
\rho\left(t_{k}\right) & =\rho_{D}, \quad \Delta \rho\left(t_{k}\right)=0 \quad \text { in the sense of } L^{2}\left(\Gamma_{D}\right) .
\end{aligned}
$$


Combining all these estimates, together with the monotonicity of $A$ (see (3.3)) and Lemma 3.4 gives after summation

$$
\begin{aligned}
\frac{1}{2}\left\|e_{k}\right\|_{L^{2}(\Omega)}^{2}+M \varepsilon^{2} \sum_{l=1}^{k} \tau_{l}\left\|e_{k}\right\|_{W^{m, p}(\Omega)}^{2} \leq \frac{1-\eta}{\eta} & \sum_{l=1}^{k} \int_{\Omega}\left(\rho_{t}\left(t_{l}\right) \tau_{l}+f_{l}\right)^{2} \mathrm{~d} x \\
& +2 \sum_{l=1}^{k}\left\|f_{l}\right\|_{L^{2}(\Omega)}^{2}+2 c_{0} \sum_{l=1}^{k} \tau_{l}\left\|\nabla\left(V\left(t_{l}\right)-V_{l}\right)\right\|_{L^{6}(\Omega)}\left\|e_{l}\right\|_{L^{2}(\Omega)},
\end{aligned}
$$

where $M=M(\Omega, \delta)>0$ is the constant specified in Lemma 3.5. Estimating

$$
\left\|f_{k}\right\|_{L^{2}(\Omega)}^{2} \leq \tau_{k}^{2}\left\|\rho_{t t}\right\|_{L^{2}\left(\Omega \times\left(t_{k-1}, t_{k}\right)\right)}^{2},
$$

and

with $c_{1}=c_{1}(\Omega, \lambda)>0$, yields

$$
\left\|\nabla\left(V\left(t_{k}\right)-V_{k}\right)\right\|_{L^{6}(\Omega)} \leq c_{1} \delta^{-1}\left\|e_{k}\right\|_{L^{2}(\Omega)},
$$

$$
\begin{aligned}
\frac{1}{2}\left\|e_{k}\right\|_{L^{2}(\Omega)}^{2}+M \varepsilon^{2} \sum_{l=1}^{k} \tau_{l}\left\|e_{k}\right\|_{W^{m, p}(\Omega)}^{2} \leq & c_{2} \sum_{l=1}^{k} \tau_{l}^{2}\left(\left\|\rho_{t}\right\|_{L^{\infty}\left(t_{l-1}, t_{l} ; L^{2}(\Omega)\right)}^{2}+\left\|\rho_{t t}\right\|_{L^{2}\left(\Omega \times\left(t_{l-1}, t_{l}\right)\right)}^{2}\right) \\
& +2 c_{0} c_{1} \delta^{-1} \sum_{l=1}^{k} \tau_{l}\left\|e_{l}\right\|_{L^{2}(\Omega)}^{2}
\end{aligned}
$$

where $c_{2}=c_{2}(\delta)>0$. Choose $\tau_{0}<\frac{\delta^{2}}{4 c_{1}}$. Then

$$
\left(\frac{1}{2}-2 c_{1} \delta^{-2} \tau_{0}\right)\left\|e_{k}\right\|_{L^{2}(\Omega)}^{2}+M \varepsilon^{2} \sum_{l=1}^{k} \tau_{l}\left\|e_{k}\right\|_{W^{m, p}(\Omega)}^{2} \leq c_{2}\|\rho\|_{H^{2}\left(0, T ; L^{2}(\Omega)\right)}^{2} \tau^{2}+2 c_{0} c_{1} \delta^{-1} \sum_{l=1}^{k-1} \tau_{l}\left\|e_{l}\right\|_{L^{2}(\Omega)}^{2} .
$$

Now it follows from the discrete Gronwall lemma that

$$
\left\|e_{k}\right\|_{L^{\infty}\left(L^{2}\right)}^{2}+M \varepsilon^{2}\left\|e_{k}\right\|_{L^{2}\left(W^{m, p}\right)}^{2} \leq c_{3} \mathrm{e}^{a t_{k}} \tau^{2}
$$

for some $c_{3}, a>0$. The estimates on $F^{\tau}-F$ and $V^{\tau}-V$ follow immediately from standard results of elliptic theory.

Remark 3.6. Although we do not get an estimate on $\rho^{\tau}-\rho$ in $L^{2}\left(0, T, H^{2}(\Omega)\right)$, the regularity in space is by far sufficient to define a suitable finite element discretization of (1.4).

\section{Conclusions}

In this paper we analyzed a new semidiscretization for a nonlinear fourth order parabolic system. The numerical scheme exploits the special nonlinear structure of the equations and its convergence strongly depends on some monotonicity property of the nonlinearity. In contrast to the nonlinear entropy methods employed in the numerical analysis of lubrication-type equations, the positivity of the scheme is not related to the degeneracy of the nonlinearity. Future work will focus on semi-implicit schemes which allow for an adequate handling of the nonlinear elliptic systems. 
Acknowledgements. The authors acknowledge financial support from the TMR Project 'Asymptotic Methods in Kinetic Theory', grant number ERB-FMBX-CT97-0157. The first author was supported by the Deutsche Forschungsgemeinschaft, grant JU 359/3 (Gerhard-Hess Program) and grant JU 359/5 (Priority Program "Multi-scale Problems"), and by the AFF project of the University of Konstanz, grant 4/00.

\section{REFERENCES}

[1] R.A. Adams, Sobolev Spaces. First edition, Academic Press, New York (1975).

[2] M.G. Ancona, Diffusion-drift modelling of strong inversion layers. COMPEL 6 (1987) 11-18.

[3] J. Barrett, J. Blowey and H. Garcke, Finite element approximation of a fourth order nonlinear degenerate parabolic equation. Numer. Math. 80 (1998) 525-556.

[4] N. Ben Abdallah and A. Unterreiter, On the stationary quantum drift diffusion model. Z. Angew. Math. Phys. 49 (1998) $251-275$.

[5] F. Bernis and A. Friedman, Higher order nonlinear degenerate parabolic equations. J. Differential Equations 83 (1990) 179-206.

[6] A.L. Bertozzi, The mathematics of moving contact lines in thin liquid films. Notices Amer. Math. Soc. 45 (1998) 689-697.

[7] A.L. Bertozzi and M.C. Pugh, Long-wave instabilities and saturation in thin film equations. Comm. Pure Appl. Math. 51 (1998) 625-661.

[8] A.L. Bertozzi and L. Zhornitskaya, Positivity preserving numerical schemes for lubriaction-typeequations. SIAM J. Numer. Anal. 37 (2000) 523-555.

[9] P.M. Bleher, J.L. Lebowitz and E.R. Speer, Existence and positivity of solutions of a fourth-order nonlinear PDE describing interface fluctuations. Comm. Pure Appl. Math. 47 (1994) 923-942.

[10] W.M. Coughran and J.W. Jerome, Modular alorithms for transient semiconductor device simulation, part I: Analysis of the outer iteration, in Computational Aspects of VLSI Design with an Emphasis on Semiconductor Device Simulations, R.E. Bank Ed. (1990) 107-149.

[11] R. Dal Passo, H. Garcke and G. Grün, On a fourth-order degenerate parabolic equation: Global entropy estimates, existence and quantitative behavior of solutions. SIAM J. Math. Anal. 29 (1998) 321-342.

[12] C.L. Gardner, The quantum hydrodynamic model for semiconductor devices. SIAM J. Appl. Math. 54 (1994) $409-427$.

[13] C.L. Gardner and Ch. Ringhofer, Approximation of thermal equilibrium for quantum gases with discontinuous potentials and applications to semiconductor devices. SIAM J. Appl. Math. 58 (1998) 780-805.

[14] I. Gasser and A. Jüngel, The quantum hydrodynamic model for semiconductors in thermal equilibrium. Z. Angew. Math. Phys. 48 (1997) 45-59.

[15] I. Gasser and P.A. Markowich, Quantum hydrodynamics, Wigner transform and the classical limit. Asymptot. Anal. 14 (1997) $97-116$.

[16] G. Grün and M. Rumpf, Nonnegativity preserving convergent schemes for the thin film equation. Numer. Math. 87 (2000) $113-152$

[17] M.T. Gyi and A. Jüngel, A quantum regularization of the one-dimensional hydrodynamic model for semiconductors. Adv. Differential Equations 5 (2000) 773-800.

[18] A. Jüngel, Quasi-hydrodynamic Semiconductor Equations. Birkhäuser, PNLDE 41 (2001).

[19] A. Jüngel and R. Pinnau, Global non-negative solutions of a nonlinear fourth order parabolic equation for quantum systems. SIAM J. Math. Anal. 32 (2000) 760-777.

[20] A. Jüngel and R. Pinnau, A positivity preserving numerical scheme for a nonlinear fourth-order parabolic system. $S I A M$ J. Numer. Anal. 39 (2001) 385-406.

[21] P.A. Markowich, Ch. A. Ringhofer and Ch. Schmeiser, Semiconductor Equations. First edition, Springer-Verlag, Wien (1990).

[22] F. Pacard and A. Unterreiter, A variational analysis of the thermal equilibrium state of charged quantum fluids. Comm. Partial Differential Equations 20 (1995) 885-900.

[23] P. Pietra and C. Pohl, Weak limits of the quantum hydrodynamic model. To appear in Proc. International Workshop on Quantum Kinetic Theory.

[24] R. Pinnau, A note on boundary conditions for quantum hydrodynamic models. Appl. Math. Lett. 12 (1999) $77-82$.

[25] R. Pinnau, The linearized transient quantum drift diffusion model - stability of stationary states. $Z A M M$ 80 (2000) $327-344$.

[26] R. Pinnau, Numerical study of the Quantum Euler-Poisson model. To appear in Appl. Math. Lett.

[27] R. Pinnau and A. Unterreiter, The stationary current-voltage characteristics of the quantum drift diffusion model. SIAM J. Numer. Anal. 37 (1999) 211-245.

[28] J. Simon, Compact sets in the space $L^{p}(0, T ; B)$. Ann. Mat. Pura Appl. 146 (1987) 65-96.

[29] G.M. Troianiello, Elliptic Differential Equations and Obstacle Problems. First edition, Plenum Press, New York (1987). 